\title{
Conf-890708--7
}

ire vommed menucripl nes been enctiored by a comrector of the U.S.

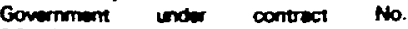
DE-ACOS-84OA2 1400 . Nocordingty. the U.S. Governinam retrine nomexcheive.

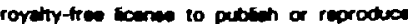
the pubcithed form of thin contriturion. Ullow ottere to do so. Io U.S. Government purpoene.-

\section{SYNCHROTRON-RADIATION EXPERIMENTS WITH RECOIL IONS}

\author{
Jon C. Levin
}

Department of Physics, University of Tennessee, Knoxville, Tennessee 37996-1200

and Oak Ridge National Laboratory, Oak Ridge, Tennessee 37831-6377

Studies of atoms, ions and molecules with synchrotron radiation have generally focused on measurements of properties of the electrons ejected during, or after, the photoionization process. Much can also be learned, however, about the atomic or molecular relaxation process by studies of the residual ions or molecular fragments following inner-shell photoionization. Measurements are reported of mean kinetic energies of highly charged argon, krypton, and xenon recoil ions produced by vacancy cascades following inner-shell photoionization using white and monochromatic synchrotron $x$ radiation. Energies are much lower than for the same charge-state ions produced by charged-particle impact. The results may be applicable to design of future angle-resolved ionatom collision experiments. Photoion charge distributions are presented and compared with other measurements and calculations. Related experiments with synchrotron-radiation produced recoil ions, including photoionization of stored ions and measurement of shakeoff in near-threshold excitation, are briefly discussed.

\section{INTRODUCTION}

Beams of low-energy, high-charge-state ions, typically formed from recoils produced by swift heavy-ion impact, have long been used to study charge-changing reactions with gas targets. In recent years, interest in the fundamental process of electron transfer in atomic collisions, in ion-atom collisions in cold interstellar clouds, and in the spectroscopy of multiply charged ions, has resulted in ever-lower center-of-mass energies. Production of highly ionized and excited ions at low energies poses a difficult challenge, since typical sources of such ions, such as stellar, fusion, and laser plasmas typically involve energies in the 1 to $100 \mathrm{keV}$ energy range. ${ }^{1}$ Fastbeam sources which achieve similar ionization-excitation states typically involve beam velocities $v / c \approx 0.1 .^{2}$ In both cases, Doppler broadening limits spectroscopic precision. Similarly, the emittance of plasma and fast beam sources is not suited for high-charge state ion-atom collision experiments at $\mathrm{eV}$ to $\mathrm{keV}$ energies under conditions where good energy and angular definition of high-chargestate projectile ions are important.

Use of fast, heavy-ion impact on target atoms to produce excited, high-charge state recoil ions ("hammer" method) permits achievement of four orders of magnitude lower energy than plasma sources in which similar ionization states have comparable abundance, as well as three orders of magnitude in $v / c$ relative to fast beam sources. As beam energies are pushed lower, ${ }^{3}$ the inherent recoil energy resulting from the primary ionization may begin to limit the resolution of the low-energy secondary beam. Our measurements of energies of recoil ions indicate that highly charged ions produced by synchrotron radiation have kinetic energies one to two orders of magnitude less than do similar charge states of recoil ions produced by charged-particle impact, providing up to six orders of magnitude lower energies and four orders of magnitude advantage in $v / c$ compared to plasms sources. The results may be applicable to development of a very-cold ion source featuring low energy spread and good angular definition.

Use of synchrotron radiation to produce a low emittance, sub-nanosecond pulsed ion source may permit improved coincidence experiments in such areas as:

- angle-resolved high-charge-state ion-atom and ion-molecule collisions in the few eV to several hundred eV range;

- interaction of tunable monochromatic radiation simulating stellar radiation with cold ions and molecules such as are found in interstellar clouds;

o precision spectroscopy of few-electron ions, following trapping and further cooling made easier by production of already cold ions;

- study of low-energy ion-surface and moleculesurface interactions. 
In recent years, development of electron cycloctron resonapce (ECR) ion sources, and a new generation of cryogenic electron-beam ion sources (such as CRYSIS at MSI in Stockholm and CRYEBIS at Kansas State's MacDonald Laboratory) has resulted in production of usable numbers of ions in very-high charge states. The high charge states accessible with such machines cannot be produced by creation of a single inner-shell vacancy, where the charge state reached is limited by the subsequent vacancy cascade, including shakeoff processes. Existing synchrotron-radiation sources are not sufficiently intense to permit multiple ionization without storage of the ions in the photon beam. An experimental program aimed at observing sequential inner-shell photoionization of ions stored in a Penning trap will be discussed.

\section{RECOIL ENERGIES}

Synchrotron $x$ radiation from the Stanford Synchrotron Radiation Laboratory (SSRL) was used to produce $K$-shell vacancies in neon and argon, and L-shell vacancies in krypton and xenon. The subsequent vacancy cascades produced multiply charged ions which were detected by time-of-flight (TOF) techniques. Examination of the resultant charge-state peaks permitted extraction of information about the mean kinetic energy of each charge state.

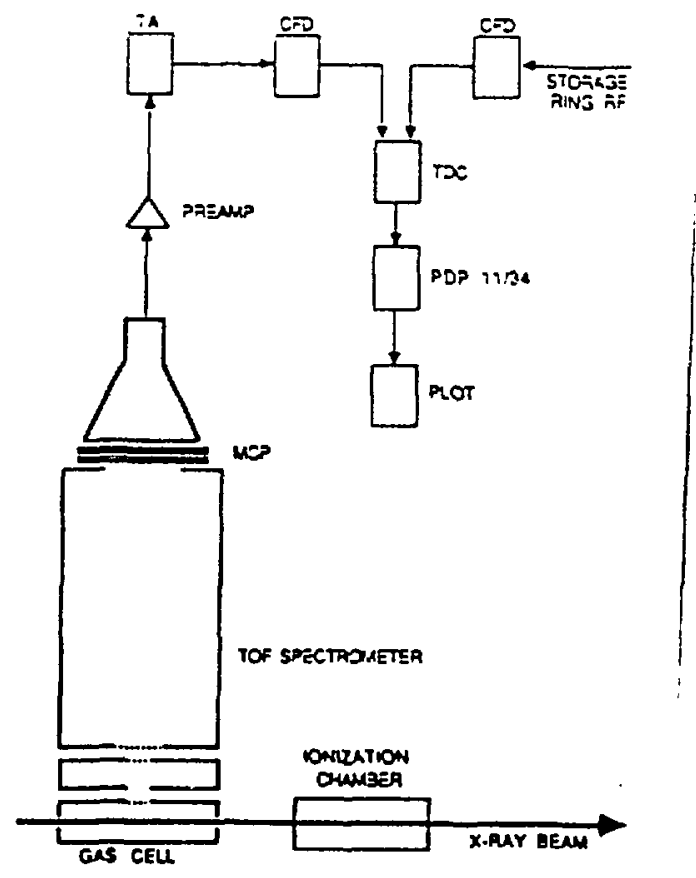

FIG. 1. Schematic of time-of-flight apparatus and associated electronics used to measure recoil-ion charge distributions and mean energies.
Beams of white or monochromatic $x$ rays from an eight-pole wiggler on SSRL beam line IV-II operated at $15 \mathrm{kG}$ were focused by a toroidal mirror and collimated to a $1 \mathrm{~mm}$ diameter $\mathrm{x}$-ray beam at the position of a thin gas target $(\approx 0.2 \mathrm{mTorr})$. The critical energy of the radiation was $4 \mathrm{keV}$ (corresponding to the 2 $\mathrm{GeV}$ electron-beam energy) and was attenuated below 3 $\mathrm{keV}$ by beryllium windows. The gas target was viewed by a vertically mounted TOF anaylzer through a $3-\mathrm{mm}$ aperture located just above the $\mathrm{x}$-ray beam. X-ray intensity was monitored by ion chambers positioned both upstream and downstream of the vacuum system housing the analyzer. Typical flux through the target was $\approx 10^{12}$ photons $\mathrm{mm}^{-2} \mathrm{~s}^{-1}$. The TOF analyzer was designed so that ion flight times were less than the $780 \mathrm{~ns}$ between bursts of photons characteristic of SSRL timing mode operation. Each pulse of light had a measured full width at half maximum (FWHM) of $\approx 300$ picoseconds 4 and was synchronous with a start signal to a CAMAC time-to-digital converter (TDC). Ions detected by a dual channel-plate detector after traversing the $\approx 10 \mathrm{~cm}$ analyzer provided the stop signal to the TDC. Timing resolution was better than 1 ns, due to the stability of the fast-rise-time TOF start pulses derived from the storage-ring if electronics, and to lownoise time-pickoff techniques applied to pulses from the detector. Typical counts rates were from 1 to $5 \mathrm{kHz} .^{5}$ The apparatus used is shown schematically in Fig. 1.

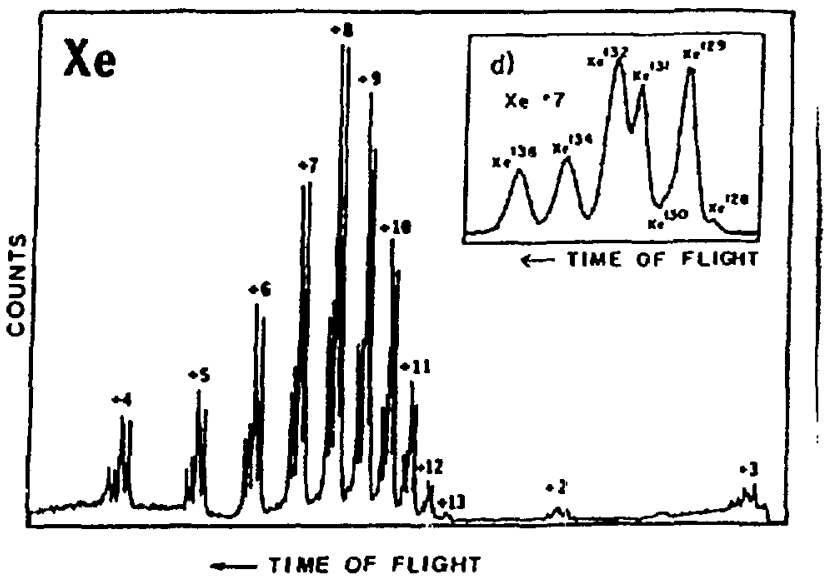

FIG. 2. Xenon time-of-flight spectrum obtained with unmonochromatized synchrotron radialion. The charge distribution is the result of a single vacancy created, most frequently, in the $L$ skell. Expanded view of $X e^{7+}$ shows resolution of renon isotopes. Peak widths are $\approx 2.2$ nsec. From ref. 6. 
The timing resolution was sufficient to obtain adjacent-mass isotopic resolution of xenon photoionized, primarily in the $\mathrm{L}$ shell, by unmonochromatized synchrotron radiation (Fig. 2). This fact is qualitative indication of low ion kinetic energy -otherwise the TOF spectra would be smeared. The peaks shown for $\mathrm{Xe}^{7+}$ in Fig. 2 have areas corresponding ciosely to known isotopic abundances and have FWHM of $\approx 2.2$ nanoseconds.

Mean recoil-ion energies were determined for each charge state $q$ through study of the FWHM of the corresponding TOF peaks, obtained by a least-squares gradient-search fitting procedure. ${ }^{7}$ The ratios of the electric fields across the four spectrometer field regions provide for first-order focusing in flight time of ions created at different distances from the detector across the $1 \mathrm{~mm}$ diameter source region. The magnitudes of the electric fields largely determine flight time. For each charge state $q$, however, TOF peak widths are the result of several contributions. These peak widths are assumed to be the result of three contributions to variations in flight time: a constant electronic timing contribution, denoted by $\alpha$, independent of spectrometer field strength $E$ and charge q; a contribution arising from field fringing and imperfect compensation for finite source width (only first-order "time focusing" is expected) which scales as $\sqrt{(1 / q E)}$, represented by $\beta$; and a third contribution resulting from the vector velocity distribution of the ions at creation, denoted by $\gamma$. Ions of mass it $m$ originally headed away from the detector must be turned around, and consequently arrive after those originally headed towards the detector. This difference in time is

$$
(\Delta t)_{r e}=2 \sqrt{2 m U_{0}} /(q E)=\gamma /(q E),
$$

indicating that the initial energy $\left(U_{0}\right)$ of the ions produces a width contribution which scales as $1 /(\mathrm{qE}) .^{8}$ Each contribution to peak width has a different functional dependence on the product $\mathbf{q E}$; addition in quadrature of these three components results in the expression:

$$
(F W H M)^{2}=\alpha^{2}+\frac{\beta^{2}}{(q E)}+\frac{\gamma^{2}}{(q E)^{2}} .
$$

By varying the absolute magnitude of the electric fields in the extraction and acceleration regions of the spectrometer, while preserving their ratio to maintain space focusing, a set of spectra can be obtained whose peak widths (FWHM) can be fitted to the quadratic function of Eq. 2. Mean recoil-ion energy $U_{0}$ can thus be extracted from the fitted quadratic term using Eq. 1. Monte-Carlo simulations of our spectrometer using principles first outlined by Wiley and $\mathrm{McClaren}^{8}$ have confirmed the general validity of this procedure. ${ }^{9}$

The clustering of data along quadratic curves is illustrated in Fig. 3 for recoil ions $\mathrm{Ar}^{6+}, \mathrm{Kr}^{4+}$, and $\mathrm{Xe}^{8+}$, and is representative of the quadratic behavior exhibited by other charge states. The influence of quadratic terms is evident, and the near-zero intercept is consistent with electronic timing resolution better than $1 \mathrm{~ns}$. Extraction of recoil energy from fitted quadratic terms $\gamma$ using $E q$. 1 resulted in the energies summarized in Table 1.

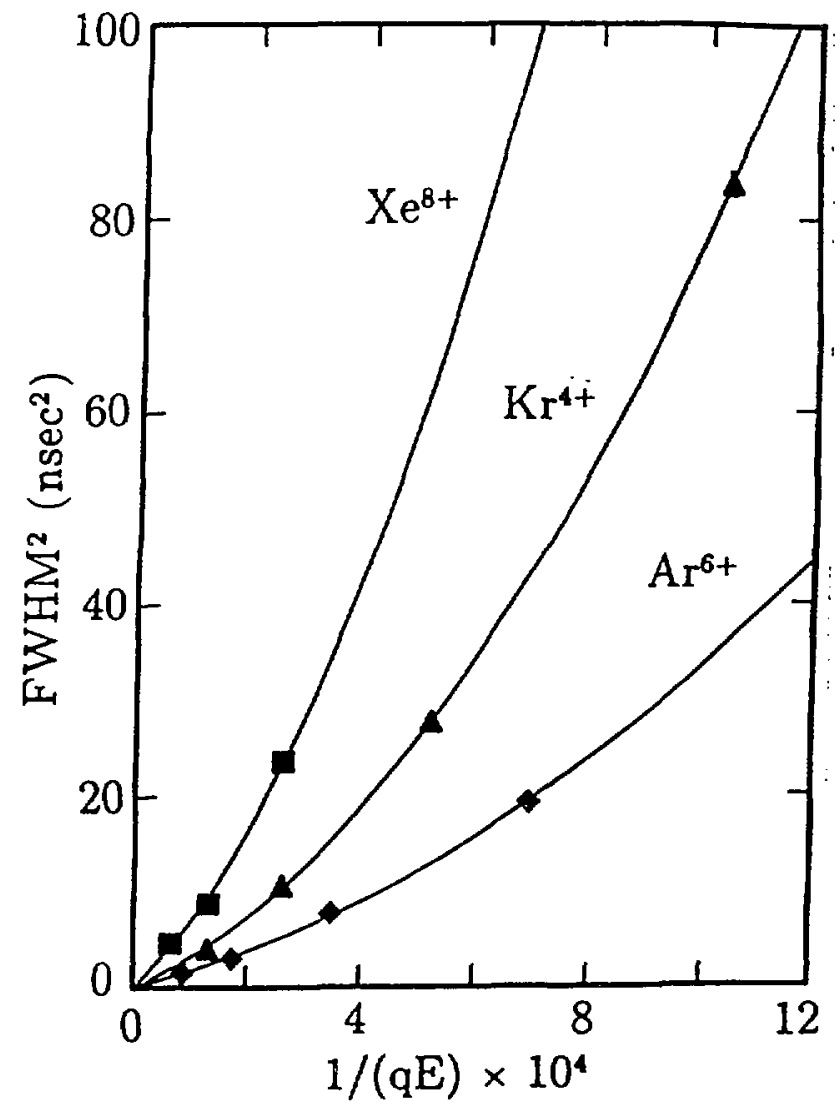

FIG. 3. Guadratic behavior of $(F W H M)^{2}$ as a function of $1 / q E$ for $A r^{6+}, K^{4+}$, and $X e^{8+}$, from which kinetic energy is extracted. 
We can make some simple estimates of kinetic energies expected of ions created by photoionization. Ion kinetic energy is the cumulative result of several effects: initial thermal energy, recoil from the photoelectron, and recoil from the several Auger and Coster-Kronig electrons ejected during the vacancy cascade which follows the primary ionization. Only velocity associated with thermal energy is isotropic. Angular momentum and parity conservation restrict the form of the angular distribution of electrons ejected by electric dipole excitation. ${ }^{10}$ The differential cross section for photoelectrons ejected from a randomly oriented target by planepolarized incident radiation, such as synchrotron radiation, is given by

$$
\frac{d \sigma(\epsilon)}{d \Omega}=\frac{\sigma(\epsilon)}{4 \pi}\left[1+\beta(\epsilon) P_{2}(\cos \theta)\right]
$$

where the Legendre polynomial $P_{2}$ is evaluated at the angle $\theta$ between the electron ejected with energy $\epsilon$ and the photon polarization vector. The $\beta(\epsilon)$ asymmetry parameter is constrained to be between -1 and +2 and oscillates strongly as a function of energy. " Similarly, the Auger-electron distribution is not isotropic and depends on photon energy. ${ }^{11}$ Since the energies in Table 1 were determined for photoionization with unmonochromatized synchroton radiation, for which photon energies range from the photoionization threshold to the $10 \mathrm{keV}$ cutoff of the focusing mirror, the direction of the recoilion velocity vector will depend in a complicated way on photon energy. Without taking these angular and energy effects, or the effect of different decay channels into account, the simple predictions of recoil energy in Table 1 were obtained by the addition, in quadrature, of thermal energy, recoil from the photoelectron produced by photons at the SSRL critical energy, and recoil from the most energetic Auger event.

The possible use of synchrotron-radiation-produced highly charged ions in low-energy ion-atom collision experiments can be illustrated by comparing the ion energies in Table 1 with energies of the same charge state ions of Ar produced by fast charged-particle beams. We have used TOF techniques similar to those already described to detect Ar recoil ions created by beams of $23 \mathrm{MeV} / \mathrm{u}$ and $27.6 \mathrm{MeV} / \mathrm{u} \mathrm{Cl}^{5+}$ produced by the Oak Ridge National Laboratory EN Tandem accelerator. ${ }^{12}$ The charged-changed projectiles, separated by charge state in a parallel-plate electrostatic field, provided the start pulse to a time-to-amplitude converter (TAC). Recoil ions detected by the TOF spectrometer stopped the TAC. High-charge-state recoil ions produced in this manner can have kinetic energies 1-2 orders of magnitude higher than when created by synchrotron radiation (Fig. 4). This is because recoil energies associated with charged-particle production are the result of the internuclear Coulomb repulsion between projectile and target, which typically are separated, at the distance of closest approach, by less than 0.5 a.u. At such small internuclear separations, near the radius of both projectile and target L-shell electrons, the effective charges due to reduced screening are much larger than the final, asymptotic charges detected experimentally. HartreeFock calculations indicate that at the distance of closest approach, 0.35 a.u. ${ }^{12}$, effective target and projectile charges for the $\mathrm{Cl}^{5+}+\mathrm{Ar} \rightarrow \mathrm{Cl}^{8+}+\mathrm{Ar}^{9+}$ system are 12.6 and 11.9 , respectively, much higher than the asymptotic charges 9 and 8 .

\begin{tabular}{|c|c|c|c|}
\hline Charge & $\operatorname{Ar}(\mathrm{K})$ & $\mathrm{Kr}(\mathrm{L})$ & $\mathrm{Xe}(\mathrm{L})$ \\
\hline 3 & $0.031(13)$ & $0.052(13)$ & \\
\hline 4 & $0.038(18)$ & $0.066(17)$ & \\
\hline 5 & $0.037(23)$ & $0.083(22)$ & $0.076(11)$ \\
\hline 6 & $0.046(29)$ & $0.094(27)$ & $0.089(14)$ \\
\hline 7 & $0.036(82)$ & $0.099(32)$ & $0.125(16)$ \\
\hline 8 & $0.023(99)$ & $0.059(34)$ & $0.121(47)$ \\
\hline 9 & & & $0.196(21)$ \\
\hline 10 & & & $0.145(23)$ \\
\hline estimate & 0.05 & 0.03 & 0.03 \\
\hline
\end{tabular}

Table 1. Kinetic energies, in eV, of $A r, K r$, and $X e$ ions produced by vacancy cascades following inner-shell vacancy creation, primarily in the shell indicated, by synchrotron-radiation photoionization. Statistical uncertainties in the last digits are indicated in parentheses.

The recoil energy produced by a charged-particle projectile depends on beam energy. As indicated by Fig. 4, slower projectiles have more time to interact with the target, producing more recoil energy. The results of Fig. 4 are consistent with data obtained at 18 $\mathrm{MeV}$ and $33 \mathrm{MeV}$, but not shown on Fig. 4. Shell effects are suggested by the much larger kinetic energies associated with production of $\mathrm{Ar}^{9+}$ in coincidence with triple-electron loss from the $\mathrm{Cl}^{5+}$ projectile. To achieve this final state, it is necessary that both projectile and target be ionized into the $L$ shell. The small impact parameters necessary to effect this degree of ionization produce the large recoil energy measured. 


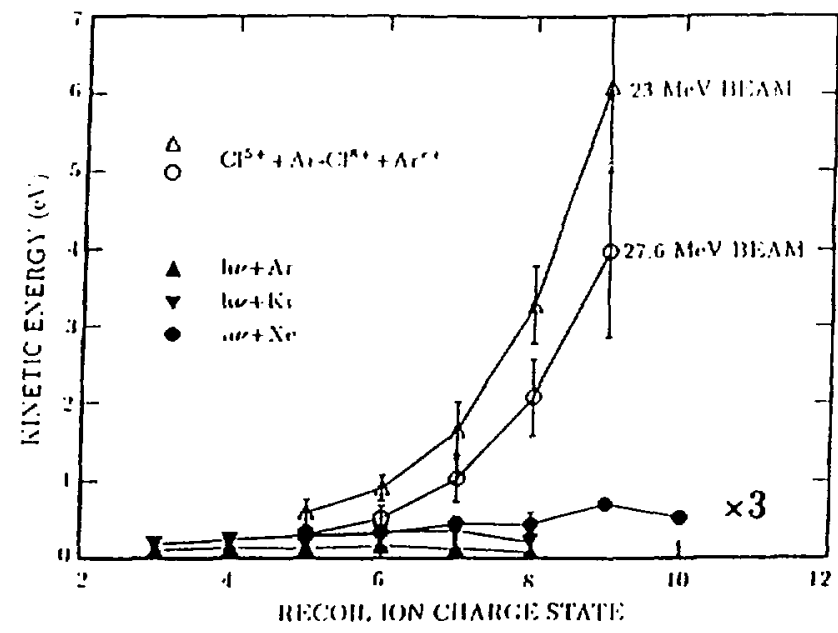

FIG. 4. Comparison of kinetic energies of Ar ions produced by charged-particle impact and by synchrotron radiation. The synchrotron-radiation data have been multiplied by 9 for clarity. From ref. 19.

\section{CHARGE DISTRIBUTIONS}

It is well known that the atomic rearrangement process following inner-shell vacancy production can lead to highly ionized states of the residual atom. The theoretical estimation of the cisarge distributions which result from this vacancy cascade must include relative cross sections for the various radiative, Auger, and Coster-Kronig processes which fill the initial, and subsequent, vacancies until the atom reaches a stable state in which no more transitions are possible. Immediately following ejection of an electron, the remaining ensemble of electrons are not in eigenstates of the ion; the sudden change in the effective charge can then result in "shake" phenomena, in which one or more electrons may be excited to unoccupied bound states ("shakeup") or into the continuum ("shakeof"). Shakeoff probabilities can be quite large, and when the effect is multiplied during a vacancy cascade, shakeoff can contribute strongly to the formation of high-charge-state ions. Following creation of a single $L_{2}$ vacancy in argon, for example, calculations show that shakeoff results in $14.8 \%$ abundance of $\mathrm{Ar}^{3+}$, and $0.2 \%$ of $\mathrm{Ar}^{4+}$; in the absence of shakeoff, the result is $100 \% \mathrm{Ar}^{2+.14}$

FIG. 5. Comparison of measured Xe charge distributions following creation of an inner-shell vacancy. The deepest shell ionized is indicated. Solid bars are data from our group and open bars are theory from ref. 16.

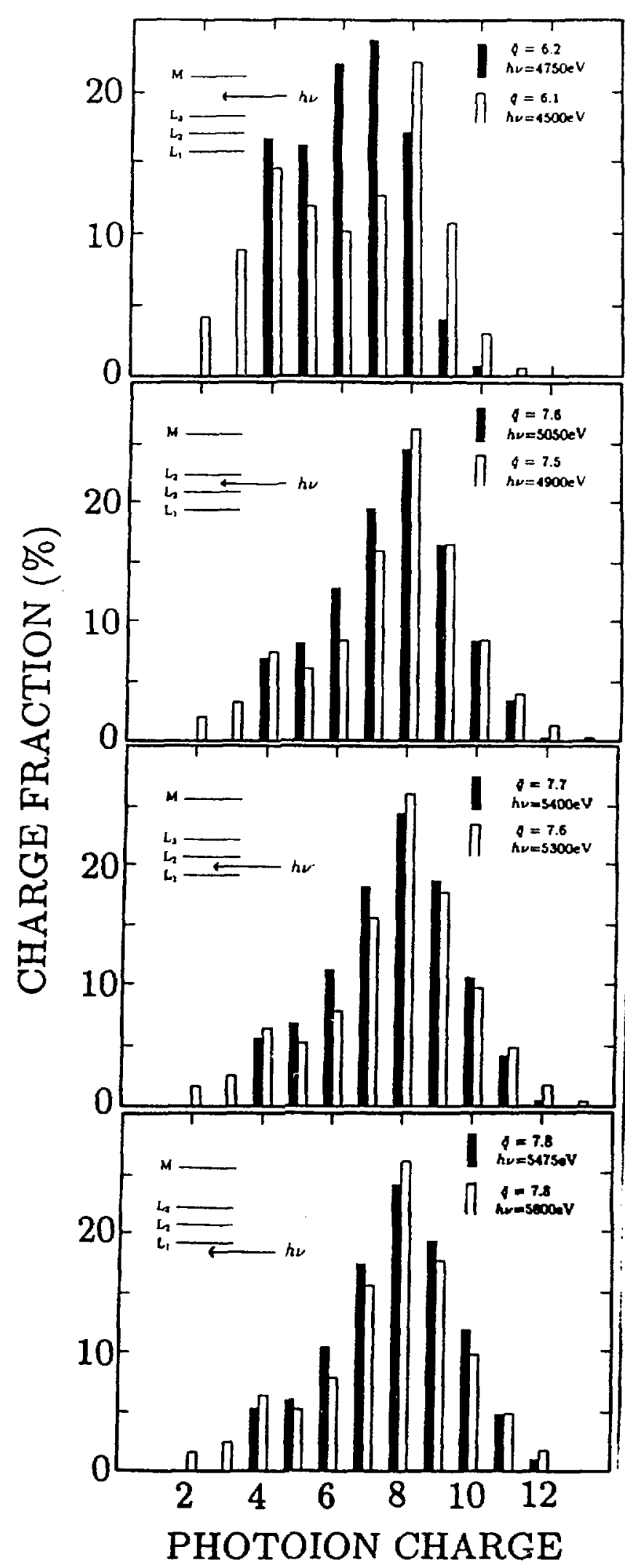


We have examined shell effects by tuning synchrotron radiation with a $S i(111)$ double-crystal mono chromator (bandpass $1 \times 10^{-4}$ ) to interleave the $L_{1}, L_{2}$, and $L_{3}$ edges of $\mathrm{Xe}$. The resultant charge distributions, which are shown in Fig. 5, can be compared with early mass-spectroscopic measurements made by Carlson et $a l,{ }^{15}$ using $x$-ray guns and filters, and recent measurements by Tonuma et a $f^{16}$ with monochromatic $x$ rays at the Photon Factory. The agreement between our results and the measurements of Tonuma et al is very good, resolving a discrepancy we observed ${ }^{5}$ with Carlson et al in the mean-charge-state increment measured for Xe as the $L_{1}$ edge is crossed. Consideration of Coster-Kronig yields $^{17}$ for transfer of an $L_{1}$ vacancy to the $L_{2,3}$ subshells and of relative photoionization cross sections of the $L_{1,2,3}$ levels ${ }^{18}$, leads to an estimated shift in mean charge state similar to that seen by our group and that of Tonuma et al.

Calculations of charge distributions following $\mathrm{L}-$ shell ionization have been performed by Tonuma et a ${ }^{16}$ using estimates of shakeof made by Carlson et $a l^{15}$ in 1966. These estimates do not include the relaxation of the atomic-electron ensemble that occurs during the vacancy cascade, the decrease in Auger transition rates which occurs because of the depletion of outer-shell electrons, or the energy dependence of shakeoff probabilities across the photoionization threshold before the asymptotic value is reached. The result of the first effect is that some Auger decays become energetically impossible as a result of the increased electron binding energies due to decreased screening as the number of inner-shell vacancies increases. Nevertheless, the agreement between measurement and theory is reasonable for the distributions following L-vacancy production, but not so good for the $\mathrm{M}$-shell results (Fig. 5.)

Our group plans to measure the threshold energy dependence of shakeoff by determining the photoion charge distribution in coincidence with Auger electrons from particular inner shells as synchrotron radiation is tuned in small increments from below the photoionization threshold up toward the asymptotic limit. This approach should complement the more usual measurements of shake phenomena made by examining photoelectron and Auger-electron satellites, ${ }^{19}$ but may offer higher count rates due to the high collection efficiency for low-energy photoions.

Studies of charge distributions following inner-shell photoionization may help elucidate more complicated multiionization phenomena in ion-atom collisions. The independent-electron-ejection model has long been employed, with substantial success, to describe multiplevacancy production in target gases by beams of ener- getic charged projectiles. In this model, whose validity requires that the collisional velocity be much larger than the orbital velocity of the electrons being ionized, electron ejection is described by a binomial distribution of outer-shell vacancies. Extension of the model to include inner-shell ionization is accomplished by introducing an independent binomial distribution for each inner shell. In most treatments, the effect of vicancy cascades following inner-shell ionization on the final recoilion charge distribution is thus neglected. We have made measurements of argon recoil-ion charge-state distributions, in coincidence with charge-changed $\mathrm{Cl}^{5+, 8+, 10+}$ projectiles at $0.7 \mathrm{MeV} / \mathrm{u}$, whose interpretation requires the assumption of Auger vacancy cascades filling target L-shell vacancies. Good agreement with the recoil-ion charge spectra cannot be obtained by assuming only the customary binomial distribution of M-shell ionization. ${ }^{20}$ Rather than losing its electrons sequentially, from the most weakly bound to the more tightly bound, the target argon atom achieves its final charge state through a superposition of outer-shell stripping with an L-shell vacancy cascade. Similar results have been reported for cxygen and fluorine projectiles at $1 \mathrm{MeV} / \mathrm{u}^{21}$

\section{SEQUENTIAL PHOTOIONIZATION}

A research program to study sequential photoionization of multiply charged ions with unmonochromatized synchrotron radiation has been initiated by $D$. A. Church of Texas A\&M University on National Synchrotron Light Source beamline X-26C. Motivations include the possible development of a high-charge-state, high-brightness ion beam with very-low energy for use in secondary ion-atom collisions. In addition, atomic inner-shell phenomena are very sensitive to relativistic and quantum-electrodynamical effects. Precision spectroscopic studies of a range of ion charges $q$ for a fixed $Z$, or of a range of isoelectronic states, could yield information on parity-nonconserving interactions ${ }^{22}$ and hyperfine structure.

The trap used at NSLS is of the Penning type, composed of a cylindrical ring electrode and two end caps shaped to produce a dominant quadrupole potential when the end caps are at zero potential and a negative potential is applied to the ring. The trap was mounted in a vertical uniform magnetic field $B \approx 0.7 T$. The target gas was pulsed into the trap in unison with the opening of a fast shutter which permitted synchrotron radiation, focused through a slot in the trap ring electrode by a platinum-coated cylindrical mirror, to enter the trap. The pulsed-gas system permitted attainment of very-low background pressures $\left(\leq 10^{-9}\right.$ Torr), thus in- 
creasing storage times. The stored ions were detected using a resonantly excited tuned circuit. ${ }^{23}$ Due to the low energies of the multiply charged ions produced, the confining well depth required was ten times shallower than for recoil ions of similar charge states produced by fast heavy-ion impact. ${ }^{24}$

Stored xenon ions in charge states as high as $q=11^{+}$have been observed with this apparatus. Note the similarity between the stored-ion charge distribution (Fig. 6) and the distribution of xenon ions created by a similar distribution of "white" photons (Fig. 2). The trapped ions show a shift to lower charge as a result of interactions between the stored thermal multicharged ion gas and the residual neutral atoms, mainly xenon, in the trap. By varying the delay time between the close of the photon shutter and the start of the detection cycle, it is possible to measure rate coefficients for electron transfer from the neutral $\mathrm{Xe}$ to $\mathrm{Xe}^{\mathrm{t}}$. Rate coefficients have been obtained for argon ${ }^{23}$ and are presented in a poster at this conference (Wed 136).

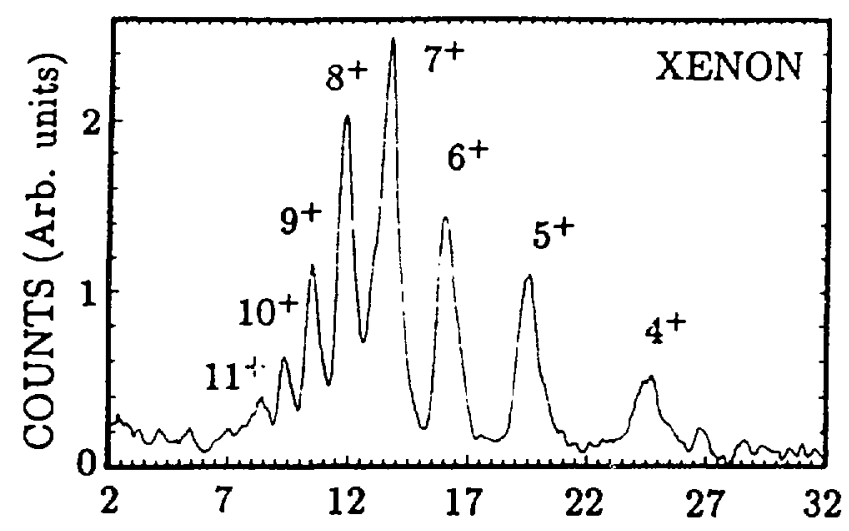

-DC RING ELECTRODE VOLTAGE

FIG. 6. Signal of multicharged Xe photoions produced by unmonochromatized synchrotron radiation and stored in a Penning ion trap. Compare with Fig. 2.

A recent measurement (June 1989) aimed at observing sequential photoionization of argon ions is undergoing analysis. Signal intensity is currently limited by decay of stored ions as a result of charge transfer and by the flux of bending-magnet radiation that passes through the beryllium windows separating the experimental chamber from ring vacuum. In the future, it may be passible to remove these windows, permitting greater flux at low energies where the cross section for $M$-shell photoionization is large.
The next generation of dedicated synchrotronradiation facilities, such as the Advanced Photon Source (APS) at Argonne and Advanced Light Source (ALS) at Berkeley, will offer many orders of magnitude greater flux than are presently available, due, in part, to increased reliance on wiggler and undulator insertion devices. These machines should make possible detailed photoionization studies of stored ions. In addition, the new high-flux light sources may permit production and extraction of highly charged photoions in sufficient quantities to form a beam useful in secondary angle-resolved ion-atom collision studies at very-low energy.

\section{Acknowledgements}

The work conducted at SSRL was performed in collaboration with R. T. Short, C.-S. O, and I. A. Sellin from the University of Tennessee (UT) and ORNL, L. Liljeby from the Manne Siegbahn Institute (MSI) in Stockholm, D. A. Church from Texas A\&M University, and S. Huldt, S.-E. Johansson, and E. Nilsson from Lund University, Lund, Sweden. The EN Tandem experiments at ORNL involved R. T. Short, C. Biedermann, C.-S. O, S. B. Elston, J. P. Gibbons, and I. A. Sellin from UT and ORNL and $H$. Cederquist, now at MSI. The Penning trap experiments at NSLS are led by D. A. Church and S. Kravis from Texas A\&M and include B. Johnson, $M$. Meron, and $K$. Jones of Brookhaven National Laboratory, R. T. Short, C.-S. O, and I. A. Sellin from UT and ORNL, G. Berry and Y. Azuma of Argonne National Laboratory, and M. Druetta of the University of Lyon.

The work of the researchers from UT/ORNL is supported in part by the National Science Foundation and b: the U.S. Department of Energy, Office of Basic Energy Sciences, Division of Chemical Sciences, under Contract No. DE-AC05-840R21400 with Martin Marietta Energy Systems, Inc. SSRL is supported by the U.S. Department of Energy, Office of Basic Energy Sciences, and the National Institutes of Health, Biotechnology Resource Program, Division of Research Resources.

\section{References}

1 E. Källne, Atomic Physics 10, 395 (1987).

${ }^{2}$ For example, see Beam Foil Spectroscopy, S. Bashkin, ed, Springer-Verlag, Berlin (1976); and Proceedings of the 1987 Symposium on Atomic Spectroscopy and Highly-lonized Atoms, H. G. Berry, ed., (1988).

${ }^{3}$ H. Cederquist, C. Biedermann, J. C. Levin, C.-S. O, I. A. Sellin and R. T. Short, Nucl. Instr. and Meth. B34, 243(1988). 
${ }^{4}$ M. G. White, R. A. Rosenberg, G. Gabon, E. D. Poli akoff, G. Thornton, S. H. Southworth, and D. A. Shirley, Rev. Sci. Instrum. 50, 1269(1979)

${ }^{5}$ R. T. Short, C.-S. O, J. C. Levin, I. A. Sellin, L. Liljeby, S. Huldt, S.-E. Johansson, E. Nilsson, and D. A. Clurch. Phys. Rev. Lett. 56, 2614 (1986).

${ }^{6}$ I. A. Sellin, J. C. Levin, C.-S. O, H. Cederquist, S. B Elston, R. T. Short, and H. Sclımidt-Böcking. Physica Scripta T22, 178(1988).

${ }^{7}$ P. Bevington, Data Reduction and Error Analysis for the Physical Sciences, McGraw-Hill, New York, 1969.

W. C. Wiley, and L.H. McLaren, Rev. Sci. Instr. 26. $1150(1955)$.

9 J. C. Levin, R. T. Short, and I. A. Sellin, to be published.

${ }^{10}$ C. N. Yang, Phys. Rev. 74, 764(1948); M. Peshkin, Adv. Chem. Phys. 18, 1(1970); V. L. Jacobs, J. Phys. B 5, 2257(1972).

${ }^{11}$ S. H. Southworth, P. H. Kobrin, C. M. Truesdale, D. Lindle, S. Owaki, and D. A. Shirley, Rapid Communications, Phys. Rev. A 24, 2257(1981).

12 J. C. Levin, R. T. Short, C.-S. O, H. Cederquisi, S. B. Elston, J. P. Gibbons, 1. A. Sellin, and H. SchmidtBöcking, Phys. Rev. A. 36, 1649(1987).

13 J. C. Levin, C. Biedermann, H. Cederquist, C.-S. O, R. T. Short, and I. A. Sellin, Nucl. Instr. and Meth. B40/41, 402(1989).

14 T. Mukoyama, Bull. Inst. Chem. Res., Kyoto Univ. $63,373(1985)$.
15 T. A. Carlson, W. E. llunt, and M. O. Krause, Phys Rev. 151, 41(1966).

16. T. Tonuma, A. Yagishita, H. Shbbata, T. Koizuma. T. Matsuo, K. Shima, T. Mukoyama, and H. Tawara, J. Phys. B20, 131(1987).

${ }^{17}$ M. II. Chen, B. Crasemann, and H. Mark, Phys. Rev A24, 177(1981).

${ }_{18}$ W. H. McMaster, N. Kerr Del Grande, J. H. Mallet, and $J$. H. Hubbel, Compilation of $x$-ray cross sections, UCRL-50174 Ser. I1 Rev. 1, Lawrence Radiation Laboratory, University of California, Livermore, 1969

${ }^{19}$ G. B. Armen, T. Ảherg, K. R. Karim, J. C. Levin, B. Crasemann, G. S. Brown, M. H. Chen, and G. E. Ice, Phys. Rev. Lett. 54, 182(1985)

${ }^{20}$ J. C. Levin, C.-S. O, H. Cederquist, C. Biedermann, and 1. A. Sellin, Rapid Communications, Phys. Rev. A 38, 2674(1988).

${ }^{21}$ O. Heber, G. Sampoll, B. B. Bandong, R. J. Maurer, E. Moler, R. L. Watson, I. Ben-Itzhak, J. L. Shinpaugh, J. M. Sanders, L. Hefner, and P. Richard, Rapid Communications, Phys. Rev. A 39, 4898(1989).

22 S. A. Blundell, W. R. Johnson, and J. Sapirstein, Phys. Rev. A 38, 4961(1988).

${ }^{23}$ D. A. Church, S. D. Kravis, I. A. Sellin, C.-S. O, J. C. Levin, R. T. Short, M. Meron, B. M. Johnson, and K. W. Jones, Rapid Communications, Phys. Rev. A 36, 2487(1987).

24 D. A. Church, R. A. Kenefick, W. S. Burns, I. A. Sellin, M. Breinig, S. B. Elston, S. Huldt, R. Holmes, J.-P. Rozet, and S. Berry, Phys. Scr. T3, 173(1983).

\section{DISCLAIMER}

This report was prepared as an account of work sponsored by an agency of the United States Government. Neither the United States Government nor any agency thsreor, nor any of their employees, makes any warranty, express or implied, or assumes any legal liability or responsibility fo: the accuracy. completeness, or usefulness of any information, apparatus, product, or process disclosed, or represents that its use would not infringe privately owned rights. Reference herein to any specific commercial product, process, or service by trade name, trademark, manufacturer, or otherwise does not necessarily constitute or imply its endorsement, recommendation, or favoring by the United States Government or any agency thereof. The views and opinions of authors expressed herein do not necessarily state or reflect those of the Urited States Government or any agency thereof. 